Sora, B., y Bohorquez, N. (2021). Convenios de doble imposición del impuesto de renta y su relación con la inversión extranjera en Colombia. Contaduría Universidad de Antioquia, 78, 13-33. Doi: https://doi. org/10.17533/udea.rc.n78a01

\title{
Convenios de doble imposición del impuesto de renta y su relación con la inversión extranjera en Colombia
}

Bibiana Marcela Sora Tibatá

Universidad Externado de Colombia

bibiana.sora@est.uexternado.edu.co

Orcid: 0000-0003-0940-5605

Nohora del Pilar Bohorquez Forero

Universidad Externado de Colombia

nohora.bohorquez@uexternado.edu.co

Orcid: 0000-0002-7100-4047 
Convenios de doble imposición del impuesto de renta y su relación con la inversión extranjera en Colombia

Resumen: La inversión extranjera directa es considerada como un factor principal en el crecimiento de la economía de los países como Colombia, ya que, permite incrementar la productividad, aumentar la innovación y fomentar el empleo; uno de los factores que determina la decisión final del inversionista es el componente fiscal, el cual se ve afectado por la doble imposición. Estudios previos afirman que los convenios de doble imposición vigentes en los países actúan como estrategia en el aumento de inversión extranjera directa, por lo tanto, a través de la metodología utilizada de mínimos cuadrados ordinarios este estudio tiene como objetivo evaluar la relación entre los convenios de doble imposición en materia del impuesto de Renta y la inversión extranjera directa en Colombia. Los resultados indican que dichos convenios no tienen ningún efecto sobre la misma. Palabras clave: Doble imposición, convenios de doble imposición, inversión extranjera directa, impuesto de renta.

Income double-tax agreements and their relationship with foreign investment in Colombia

Abstract: Foreign direct investment is considered a main factor in the economic growth of countries such as Colombia, since it allows to raise productivity, increase innovation and foster employment. One of the factors determining the final decision of the investor is the tax component, which is affected by double taxation. Previous studies argue that the current double-tax agreements in the countries act as a strategy in the increase of foreign direct investment; therefore, through the ordinary least squares methodology used, this study aims at evaluating the relationship between double-tax agreements regarding Income Tax and foreign direct investment in Colombia. The results indicate that said agreements do not have any effect on foreign direct investment.

Keywords: double taxation, double-tax agreements, foreign direct investment, income tax.

Parcerias de dupla tributação do imposto de renda e sua relação com o investimento estrangeiro na Colômbia

Resumo: $O$ investimento estrangeiro direto é considerado como um fator principal no crescimento da economia dos países como a Colômbia, já que permite incrementar a produtividade, aumentar a inovação e fomentar o emprego; um dos fatores que determina a decisão final do investidor é o componente fiscal, o que se vê afetado pela dupla tributação. Estudos prévios afirmam que as parcerias de dupla tributação vigentes nos países atuam como estratégia no aumento do investimento estrangeiro direto, por isso através da metodologia utilizada dos mínimos quadrados ordinários, este estudo tem como objetivo avaliar a relação entre as parcerias de dupla tributação em matéria de imposto de renda e o investimento estrangeiro direto na Colombia. Os resultados mostram que tais parcerias não têm nenhum efeito sobre a mesma.

Palavras chave: dupla tributação, parcerias de dupla tributação, investimento estrangeiro direto, imposto de renda.

Conventions de double imposition de l'impôt sur le revenu et leur rapport avec les investissements étrangers en Colombie

Résumé: L'investissement étranger direct est considéré comme un facteur majeur de la croissance économique de pays comme la Colombie, car il permet d'accroître la productivité et l'innovation, ainsi que de promouvoir l'emploi. L'un des facteurs déterminant la décision finale de l'investisseur est l'élément fiscal, qui est affecté par la double imposition. Des études précédentes indiquent que les conventions de double imposition en vigueur dans les pays servent de stratégie pour accroître les investissements étrangers directs, Par conséquent, cette étude a pour but d'évaluer la relation entre les conventions de double imposition en matière d'impôt sur le revenu et l'investissement étranger direct en Colombie, à l'aide de la méthodologie utilisée pour les moindres carrés ordinaires. Les résultats indiquent que ces conventions n'ont aucun effet sur l'investissement étranger en Colombie. Mots-clés: double imposition, conventions de double imposition, investissement étranger direct, impôt sur le revenu. 


\title{
Convenios de doble imposición del impuesto de renta y su relación con la inversión extranjera en Colombia
}

\author{
Bibiana Marcela Sora Tibatá y Nohora del Pilar Bohorquez Forero \\ https://doi.org/10.17533/udea.rc.n78a01
}

Primera versión recibida en noviembre de 2020 - Versión aceptada en febrero de 2021

\section{Introducción}

A partir de la década de los ochenta inicia un proceso de globalización y de apertura económica en Colombia, al igual que en la mayoría de los países latinoamericanos, lo cual contribuyó en el crecimiento de inversiones, capitales y tecnología y en el aumento de operaciones comerciales en la región. Dichas operaciones se caracterizaron por la falta de homogeneidad legislativa internacional en aspectos tributarios (Herrán Ocampo, 2000; Duque Romero, 2009).

A través del tiempo, los países desarrollados han venido acumulando capital para posteriormente invertir en países en vías de desarrollo (Herrán Ocampo, 2000), lo cual se ha visto potenciado con la globalización de América Latina y permitiendo el desarrollo de la inversión extranjera. Dentro del proceso de inversión extranjera una de las variables que más importancia ha tenido y que determina la decisión final del inversionista, es el componente fiscal, ya que una misma renta o capital resulta ser gravada más de una vez (Herrán Ocampo, 2000). La doble imposición se define como "el resultado de la aplicación de impuestos similares, en dos (o más) Estados, a un mismo contribuyente respecto de la misma materia imponible y por el mismo periodo de tiempo" (OCDE, 2010). Este problema de la doble imposición ha llevado a la necesidad de buscar soluciones multilaterales que suplan las expectativas de los inversionistas. Considerando que, sin un acuerdo específico de doble tributación, son mayores las posibilidades de recurrir a la evasión fiscal y de generar una disminución en los índices de la inversión extranjera ya que los inversionistas optan por no cumplir con sus responsabilidades tributarias y 
Sora, B., y Bohorquez, N. Convenios de doble imposición del impuesto de renta...

buscan como opción otros países para invertir, donde existan condiciones más favorables. De esta manera se analiza que la solución más viable son los convenios para evitar la doble imposición (CEDI), los cuales regulan la forma de cooperación internacional entre las dos administraciones tributarias, promueven el intercambio de información fiscal y reducen la incertidumbre del inversionista (Duque Romero, 2009).Según cifras del Country Brand Ranking 2019-2020, emitidas por la firma de consultoría Bloom Consulting (2019), Colombia obtuvo un desempeño positivo en todas las variables que miden la inversión extranjera y logró posicionarse en el top 5 de las economías más atractivas para los inversores extranjeros en América Latina, lo que representa un impulso fundamental en el desarrollo económico del país, pues se traduce en puestos de trabajo, transferencia de conocimiento y avance tecnológico (Marketers by Adlatina, 2019).Como lo muestra la tabla 1, actualmente Colombia cuenta con 11 convenios de doble imposición vigentes. Adicionalmente, existes 5 tratados de negociación en materia de impuesto sobre la renta, de los cuales cinco son con países europeos, dos con países latinoamericanos, dos con países asiáticos, uno con un país norteamericano y uno con la comunidad andina de naciones.

Tabla 1: Convenios de doble imposición en materia de impuesto sobre la renta

\begin{tabular}{lcc}
\hline \multicolumn{1}{c}{ Convenio } & Fecha vigencia & Norma \\
\hline Emiratos Árabes Unidos & No vigente & \\
Japón & No vigente & \\
Francia & No vigente & \\
Italia & No vigente & \\
Gran Bretaña & $13 / 12 / 2019$ & Ley 1939 de 2018 \\
Republica Checa & $06 / 05 / 2015$ & Ley 1690 de 2013 \\
Portugal & $30 / 01 / 2015$ & Ley 1692 de 2013 \\
India & $07 / 07 / 2014$ & Ley 1668 de 2013 \\
Corea & $03 / 07 / 2014$ & Ley 1667 de 2013 \\
México & $11 / 07 / 2013$ & Ley 1568 de 2012 \\
Canadá & $12 / 06 / 2012$ & Ley 1459 de 2011 \\
Suiza & $01 / 01 / 2012$ & Ley 1344 de 2009 \\
Chile & $22 / 12 / 2009$ & Ley 1261 de 2008 \\
España & $23 / 10 / 2008$ & Ley 1082 de 2006 \\
Comunidad Andina de Naciones & $01 / 01 / 2005$ & Decisión 578 de 1004 \\
\hline
\end{tabular}

Fuente: Elaboración propia. 
El impuesto de renta y su complementario de ganancias ocasionales es un tributo directo que se debe pagar cuando la suma de los ingresos de un contribuyente genera un incremento en el patrimonio durante un periodo fiscal (generalmente un año), este impuesto integra la renta, las ganancias ocasiones y las remesas de las personas naturales y jurídicas (Acosta Rodríguez, 2013). Esta investigación se enfoca en el impuesto sobre la renta, ya que, de los países que existen actualmente el 91,2\% lo pagan, por ende, es considerado un tributo transversal. Por otro lado, según Portafolio (2020) el impuesto de renta se consolida como el rubro más grande de los ingresos por gravámenes, por más de $\$ 70$ billones de pesos, alcanzando casi un 50\% del recaudo tributario anual en Colombia. Con esta investigación se busca determinar la relación entre los convenios de doble imposición en materia de impuesto sobre la renta y la inversión extranjera directa en Colombia. Para cumplir este objetivo los investigadores utilizaron el método planteado por Carr, Markusen y Maskun (1997) y utilizado por otros autores como: Barthel Busse y Neumayer (2010), Baker (2014), Blonigen y Davies (2004), Blonigen y Davies (2005), Blonigen, Oldenski y Sly (2014), Davies (2003) y Castillo y López (2019).Este método fue elegido ya que considera variables económicas específicas que miden el comportamiento histórico de la inversión extranjera y variables adicionales de control que evalúan los principales aspectos que afectan la decisión de localización del inversionista. Permite, además, identificar los elementos de los convenios, el comportamiento histórico de la inversión extranjera directa (IED), desde la fecha de entrada en vigor del primer convenio y el comportamiento de los principales indicadores económicos, tales como el PIB real, la apertura comercial y la mano de obra calificada (Carr et al. 1997).La presente investigación se estructura de la siguiente manera: en la primera parte se efectúa una revisión literaria de artículos académicos donde se documentan las investigaciones que estudian la relación entre los convenios de doble imposición y la inversión extranjera; en la segunda parte se plantea la metodología y la descripción de las variables; en la tercera parte se discuten los resultados estadísticos obtenidos, y en la última parte se exponen las conclusiones.

\section{Revisión de literatura}

En este estudio cuantitativo correlacional se examinó la relación entre los convenios de doble imposición (CEDI) y la inversión extranjera directa (IED) en Colombia. Los artículos académicos obtenidos en esta revisión literaria se consultaron en la base de datos Springer Nature Journals, HeinOnline, Directory of Open Access Journals y Supplemental Index de la biblioteca de la Universidad Externado de Colombia consultando las palabras clave: inversión extranjera directa, tratados tributarios y doble imposición. Los artículos identificados 
Sora, B., y Bohorquez, N. Convenios de doble imposición del impuesto de renta...

se clasificaron en dos grupos dependiendo de la metodología utilizada en los estudios. El primer grupo está conformado por los autores que dentro de su estudio empírico utilizaron el método de equilibrio general propuesto por Carr et al. (1997) y el segundo grupo está conformado por los autores que utilizaron un método experimental a partir del análisis de variables económicas.

El método de equilibrio general conocimiento-capital propuesto (CMM) por Carr et al. (1997), se enfoca en el análisis estadístico del conocimiento, donde se mide la diferencia de cualificación entre los dos países y su interacción con las demás variables. En este método se realiza una clasificación de la IED de acuerdo con su motivación (horizontal y vertical), la motivación horizontal hace referencia a los países que presentan un nivel de cualificación similar, y la vertical a los países que presentan un nivel diferente, esto con el fin de analizar la correlación de manera más objetiva (Carr et al. 1997).Los autores que emplearon este método en sus estudios fueron: Blonigen y Davies (2004), Davies (2003), Blonigen y Davies (2005), Barthel et al. (2010), Blonigen et al. (2014), Baker (2014) y Castillo y López (2019).Para comenzar, Blonigen y Davies (2004) evaluaron el efecto de los CEDI enfocándolo en la actividad de IED de Estados Unidos como país de origen frente a otros países. Su estudio se basó en una regresión lineal utilizando dos métodos, inicialmente el método de gravedad tradicional y, posterior a este, el método CMM. Incluyeron como variable de control, la antiguiedad del tratado para analizar si presentaba algún efecto significativo y concluyeron que después de 1 año de antigüedad los convenios tienen un efecto positivo creciente en los flujos de salida de IED de Estados Unidos hacia los países evaluados, lo que representa un aumento entre el $2 \%$ y el $8 \%$ por cada año adicional (Blonigen y Davies, 2004).Adicionalmente, es su estudio, Davies (2003) concluyó que los convenios tienen un efecto negativo sobre la IED; en su investigación analizó la información a partir de 4 regresiones con el método $\mathrm{CMM}$, incluyendo variables de revisión relacionadas con los convenios como: la antigüiedad, la renegociación, variables ficticias como treaty ( 1 vigente, 0 si no existe) y antiguiedad ( 1 si es nuevo, 0 si es antiguo). Finalmente, el investigador concluyó que el tiempo es parcialmente responsable de los cambios en los coeficientes de las regresiones y representa un efecto negativo entre los flujos de IED y los convenios. Además, afirma que entre los propósitos de estos últimos se encuentra la disminución de la evasión fiscal y la instauración de políticas anti-compra de tratados, factores que contribuyen en la disminución de la IED (Davies, 2003).Un análisis posterior realizado por Blonigen y Davies (2005) contrarrestó la idea planteada en su estudio anterior (Blonigen y Davies, 2004), que afirmaba que los convenios promueven los flujos IED; en este nuevo análisis evaluaron la relación entre las variables a través de dos regresiones: en la primera regresión incluyeron una variable ficticia (treaty), la cual toma el valor de 1 si el par de país cuenta con 
un tratado bilateral o 0 por el contrario. Los resultados de esta regresión indicaron una relación positiva entre los CEDI y la IED. En la segunda regresión incluyeron la información de las variables antes y después de la vigencia del convenio, los resultados en este caso arrojaron una relación negativa entre los CEDI y la IED. Con base a los datos obtenidos, Blonigen y Davies (2005) concluyeron que los convenios representan un efecto negativo en la inversión extranjera, debido a que uno de sus objetivos es disminuir la evasión fiscal, lo que afecta de forma negativa a la variable dependiente (Flujos de IED) (Blonigen y Davies, 2005).Por otro lado, Barthel et al. (2010) evaluaron a través del método CMM una muestra amplia de 30 países como origen y 105 países como anfitriones por un periodo de 26 años, reduciendo de esta manera el riesgo de resultados distorsionados por un sesgo en la muestra. Dentro del análisis, agregaron la inflación del país anfitrión como una nueva variable independiente, y a partir de la aplicación de regresiones estadísticas concluyeron que los convenios están positivamente asociados con la inversión extranjera del país anfitrión, ratificando el estudio previo realizado por Blonigen y Davies (2004). Adicional a esto, Barthel et al. (2010) en su estudio describen la importancia de que cada país analice cuidadosamente los pro y contras de la negociación de un convenio, debido a que en muchos casos los países no logran suplir los altos costos de negociación y pérdida de ingresos fiscales con la entrada de flujos de IED (Barthel et al., 2010).Del mismo modo, Blonigen et al. (2014) evaluaron bajo el método CMM, el efecto de los CEDI en la inversión extranjera de países con empresas que utilizan insumos diferenciadores y analizaron que estas empresas se encuentran más expuestas a la doble imposición por las prácticas fiscales. Para el desarrollo del estudio, aplicaron un análisis estadístico a partir de una regresión donde incluyeron la variable Diff_Input, que representa en qué medida las empresas industriales utilizan insumos heterogéneos para generar una cantidad de producción establecida. El estudio concluyó que los convenios tienen un efecto positivo en la IED en países donde las empresas utilicen mayores insumos diferenciadores en su producción, lo que representa un aumento en las ventas de 10 millones de dólares por año (Blonigen et al. ,2014).Por su parte, Baker (2014) a través del método CMM realizo una estimación por puntaje de propensión entre 30 países desarrollados pertenecientes a la OCDE y 206 países en desarrollo no pertenecientes a esta organización, en este análisis tuvo en cuenta al primer grupo como países de origen y al segundo como países anfitriones de IED. Baker (2014) dividió el análisis en un enfoque cuantitativo y cualitativo y concluyó que los convenios no tienen ningún efecto sobre los flujos de IED, dado que los países en desarrollo están disminuyendo sus tasas de retención sin necesidad de firmar un convenio, y afirma que los beneficios obtenidos por estos convenios no son suficientes para influir en las decisiones de localización 
Sora, B., y Bohorquez, N. Convenios de doble imposición del impuesto de renta...

de los inversionistas, lo cual no justifican los altos costos de entrada (Baker, 2014).Para terminar la primera agrupación metodológica, Castillo y López (2019) evaluaron el efecto de la IED entre España y 56 países a lo largo del periodo 1993-2013, teniendo en cuenta tanto la inversión extranjera en España como la inversión española en el extranjero. Con el fin de realizar un análisis estadístico por grupos, los investigadores organizaron la información y resultados en países desarrollados, en vía de desarrollo, pertenecientes a la OCDE, países de la UE-28 y algunos países latinoamericanos, más los pertenecientes a la BRICS (Brasil, Rusia, India, China y Sudáfrica).Usando como referencia el estudio realizado por Davies (2003), Castillo y López (2019) realizaron una subdivisión en tratados antiguos y nuevos, tomando como fecha base 1993, y siguiendo los lineamientos de Barthel et al. (2010), realizaron una estimación para modelos estáticos y dinámicos teniendo en cuenta el efecto de los convenios según su antiguiedad. Los investigadores concluyeron que en la primera técnica los convenios presentan un efecto positivo sobre la actividad de inversión extranjera, mientras que en la segunda estos no presentan efecto significativo en la IED. Por ende, los convenios de doble imposición presentan un efecto positivo en la inversión extranjera (Castillo y López, 2019).El segundo grupo metodológico identificado en la revisión literaria y usado por Braun (2016), Murthy y Bhasin (2015), Marques y Pinho (2014) y Neumayer (2007) fue el del método experimental, el cual consiste en un análisis correlacional de los flujos de inversión extranjera y los principales indicadores económicos tales como el PIB, apertura comercial, inflación, entre otros. A diferencia del método anterior, no se tiene en cuenta variables que miden la diferencia de cualificación entre los dos países, por ende, no se tiene en cuenta la clasificación por las motivaciones de la IED.Braun (2016) evaluó la relación de los tratados de doble imposición y la IED mediante un método logístico (modelo de respuesta binaria tomando la variable dependiente como una variable ficticia), y enfocó su análisis en países en vía de desarrollo como anfitriones y Austria como país de origen. En esta investigación Braun (2016) hizo énfasis en las otras alternativas que ofrece Austria para eliminar la doble imposición, tales como exenciones o acuerdos bilaterales, afirmando que es importante que el país anfitrión logre compensar la pérdida de ingresos fiscales con el incremento de IED. Dentro de su método experimental, Braun (2016) incluyó variables independientes que miden el crecimiento económico del par de países (producto interno bruto o PIB, apertura y costos comerciales) y variables de control tales como las tasas impositivas, el índice de corrupción y la calidad de infraestructura. El estudio concluyó que la inversión extranjera aumenta de un $25 \%$ a un $33,7 \%$ en países en vía de desarrollo que cuentan con convenios de doble imposición vigentes, sin embargo, asegura que este aumento puede estar dado por otras variables no observadas y que el método 
utilizado solo mide efectos en el corto plazo ( 3 años después de la fecha de vigencia), dejando de lado los efectos de los convenios en el largo plazo (Braun, 2016).Por su parte Murthy y Bhasin (2015), examinaron la relación de los convenios con la inversión extranjera en India, utilizando como método experimental el análisis de las variables económicas tales como (PIB), PIB per cápita, apertura comercial y la variable ficticia treaty planteada anteriormente por Blonigen y Davies (2005). En principio Murthy y Bhasin (2015) realizaron un análisis estadístico teniendo en cuenta la existencia o no de un tratado de doble imposición; posteriormente incluyeron la variable de antiguiedad para inferir si el tiempo de vigencia afecta la relación de manera significativa. A partir de esto, concluyeron que la antigüiedad del convenio interactúa positivamente dentro de la relación de las variables y que el PIB per cápita se relaciona directamente proporcional con la IED (Murthy y Bhasin, 2014).Así mismo, Marques y Pinho (2014) evaluaron el efecto de los tratados en la IED, utilizando un método experimental enfocado en el análisis de las multinacionales europeas, considerando el número de nuevas filiales como variable dependiente y como variables independientes el tipo de impuestos de la matriz, la retención de impuestos de no residentes en el país anfitrión y la tasa de crecimiento del PIB. El estudio concluyó que los tratados fiscales afectan positivamente la inversión extranjera y que las tasas de impuestos del país anfitrión desempeñan un papel fundamental dentro de esta relación. Lo anterior indica que un aumento en un punto porcentual impositivo genera una disminución en promedio de 9 filiales y la presencia de un tratado aumenta en un 27\% la entrada de nuevas filiales (Marques y Pinho, 2014). Finalmente, un estudio realizado por Neumayer (2007) evaluó datos de la inversión extranjera de Estados Unidos en países en vía de desarrollo durante el periodo de 19702001. Dentro de su análisis contempló los principales indicadores económicos tales como el PIB, la apertura comercial y la inflación, como variables independientes y el alquiler de recursos y adhesión a la OMC como variables de control. De esta manera, concluyó que los países en desarrollo que firman un tratado con Estados Unidos se benefician de una mayor participación en la IED; sin embargo, cuando la muestra se divide en países de ingresos bajos y medianos, los convenios solo son eficaces en el grupo de ingresos medios (Neumayer, 2006).Para resumir la aproximación literaria del estudio se presenta la tabla 2 , donde se puede observar un resumen general de la literatura empírica expuesta anteriormente; clasificada por método, autor, muestra y resultados. 
Sora, B., y Bohorquez, N. Convenios de doble imposición del impuesto de renta...

Tabla 2: Resultados de la literatura empírica por método

\begin{tabular}{|c|c|c|}
\hline Autores y años & Muestra & Resultados \\
\hline \multicolumn{3}{|l|}{ Método CMM (1997): } \\
\hline Baker (2014) & $\begin{array}{l}30 \text { países desarrollados de la OCDE y } 206 \\
\text { países en desarrollo: } 1991 \text { - } 2006\end{array}$ & Sin efecto \\
\hline Barthel et al. (2010) & $\begin{array}{l}30 \text { países origen ( } 10 \text { en desarrollados) y } 105 \\
\text { países anfitriones ( } 84 \text { en desarrollo): 1978-2004 }\end{array}$ & Positivo \\
\hline Blonigen y Davies (2004) & EE. UU: 1966-1992 & Positivo \\
\hline Blonigen y Davies (2005) & OCDE: $1982-1992$ & Negativo \\
\hline Blonigen et al. (2014) & EE. UU: $1987-2007$ & $\begin{array}{l}\text { Positivo en países con } \\
\text { mayores productos } \\
\text { diferenciadores }\end{array}$ \\
\hline Castillo y López (2019) & $\begin{array}{l}56 \text { países divididos en: desarrollados, en } \\
\text { desarrollo, OCDE, UE-28 y latinoamericanos + } \\
\text { BRICS: 1993- } 2013\end{array}$ & Positivo \\
\hline $\begin{array}{l}\text { Davies (2003) } \\
\text { Método experimental: }\end{array}$ & EE. UU: 1996-2000 & Negativo \\
\hline Braun (2016) & Austria y 104 países en desarrollo: 1990-2011 & Positivo \\
\hline Marques y Pinho (2014) & 37 países europeos: $2000-2009$ & Positivo \\
\hline Murthy y Bhasin (2015) & India: 1993-2011 & Positivo \\
\hline Neumayer (2007) & Países en desarrollo: 1970-2001 & $\begin{array}{l}\text { Positivos países con } \\
\text { ingresos medios }\end{array}$ \\
\hline
\end{tabular}

Fuente: Elaboración propia.

Actualmente existen pocos estudios que estimen los efectos de los CEDI y la IED; las evidencias se encuentran divididas en tres premisas: efecto positivo, efecto negativo y sin ningún efecto. Por lo tanto, existen opiniones contradictorias entre la literatura existente y es necesario realizar más investigaciones; investigar para determinar la verdadera relación entre las variables. Es importante señalar que ningún estudio previo encontrado en la revisión literaria ha evaluado el efecto de estos convenios en la inversión extranjera de los países latinoamericanos, y especialmente en Colombia. La mayoría de estos estudios abarcan países desarrollados, países europeos o países pertenecientes a organizaciones internacionales, como es el caso de la OCDE.Un estudio de este tipo es importante para un país como Colombia, puesto que la inversión extranjera se ha constituido como un activo fundamental en el desarrollo y crecimiento de la economía (Santoro, 2019). Esto se ha visto reflejado en la firma de diversos convenios de doble tributación relacionados con el impuesto de renta por parte de Colombia como los son: España (2008), Chile (2009), Suiza (2012), Canadá (2012), México (2013), 
Corea del Sur (2014), India (2014), Portugal (2015), Republica Checa (2015) y Gran Bretaña (2019) (DIAN, 2019).Además, uno de los beneficios adicionales a la eliminación de la doble imposición es la búsqueda de la seguridad fiscal reduciendo la incertidumbre del inversionista, lo que incentiva en gran medida a la IED, promoviendo el intercambio de información fiscal entre las dos administraciones tributarias y contribuyendo en la disminución de la evasión fiscal del impuesto de renta (Procolombia, 2019).Por consiguiente, analizando los efectos de los convenios de doble imposición, Colombia puede mejorar el modelo de negociación, replanteando si la firma de un convenio relacionado con el impuesto de renta realmente representa mayores beneficios o por el contrario son mayores costos para el país. Teniendo en cuenta lo anterior, este trabajo de investigación plantea la siguiente hipótesis:

Ho: Los convenios de doble imposición del impuesto sobre la renta aumentan la inversión extranjera directa en Colombia.

\section{Metodología}

El método utilizado en esta investigación cuantitativa correlacional es el de equilibrio general conocimiento-capital propuesto por Carr et al. (1997). Dicho método evalúa las 2 motivaciones de la IED: horizontal y vertical. Markusen (1984, citado en Castillo y López, 2019) menciona la motivación horizontal como la replicación de la actividad productiva en estados con características similares. Helpman (1984) define la motivación vertical como la replicación de la actividad productiva en estados con diferencias de cualificación con el fin de ahorrar costos (Castillo y López, 2019). Con el fin de comprobar la hipótesis se formuló la siguiente ecuación con base al método de Carr et al. (1997):

$$
I E D_{b}=f\left(S_{U M P I B}, \text { DIFFPIB2 }_{a b}, \text { DIFFHAB }_{a b}, \text { DIFFPIBHAB }_{a b}, \text { DIST, T_OPEN }\right)
$$

La variable dependiente $\left(I E D_{b}\right)$ son los datos de inversión extranjera directa del país anfitrión, en este caso Colombia, los cuales fueron obtenidos de los reportes publicados por el Banco de la República desde el año 2008 al 2019. Para identificar los datos del país de origen de inversión se asignó la letra a y para el país anfitrión la letra b. Adicionalmente a la variable dependiente, las variables independientes y sus definiciones se encuentran en la tabla 3.

Tabla 3: Descripción de las variables Método CMM

\begin{tabular}{lll}
\hline \multicolumn{2}{l}{$\begin{array}{l}\text { Descripción variables método CMM } \\
\mathrm{a}=\text { País de origen }\end{array} \quad \mathrm{b}=$ País anfitrión } \\
\hline \multicolumn{2}{l}{ Variable dependiente } \\
\hline Variables & Descripción & Fuente \\
\hline IEDb & $\begin{array}{l}\text { Flujos de Inversión extranjera directa del } \\
\text { país anfitrión }\end{array}$ & Indicadores IED Banco de la Republica \\
\hline
\end{tabular}


Sora, B., y Bohorquez, N. Convenios de doble imposición del impuesto de renta...

\begin{tabular}{|c|c|c|}
\hline \multicolumn{3}{|c|}{ Variables Independientes } \\
\hline Variables & Descripción & Fuente \\
\hline SUMPIB (log) & Suma del PIB real de los 2 países & $\begin{array}{l}\text { Indicadores de desarrollo del Banco } \\
\text { Mundial }\end{array}$ \\
\hline DIFFPIB2 (log) & $\begin{array}{l}\text { Diferencia de los PIB reales de los } 2 \text { países } \\
\text { al cuadrado (valor absoluto) }\end{array}$ & $\begin{array}{l}\text { Indicadores de desarrollo del Banco } \\
\text { Mundial }\end{array}$ \\
\hline DIFFHAB & $\begin{array}{l}\text { Diferencia de las habilidades de la } \\
\text { población de los } 2 \text { países. Esta variable } \\
\text { esta medida como el porcentaje de la } \\
\text { población inscrita en el nivel terciario de } \\
\text { educación }\end{array}$ & $\begin{array}{l}\text { Indicadores de desarrollo del Banco } \\
\text { Mundial- Institución de Estadística } \\
\text { de la Organización de las Naciones } \\
\text { Unidas para la Educación, la Ciencia y } \\
\text { la Cultura (UNESCO). }\end{array}$ \\
\hline $\begin{array}{l}\text { DIFFPIBHAB }(\log ) \\
\text { (DIFFHAB* } \\
\text { DIFFPIB) }\end{array}$ & $\begin{array}{l}\text { Es la interacción entre la diferencia de } \\
\text { las habilidades y la diferencia de los PIB } \\
\text { reales. Respecto a la diferencia de los } \\
\text { PIB se toma el del país origen - el país } \\
\text { anfitrión }\end{array}$ & $\begin{array}{l}\text { Indicadores de desarrollo del Banco } \\
\text { Mundial }\end{array}$ \\
\hline DIST (log) & $\begin{array}{l}\text { Distancia medida en millas desde la } \\
\text { capital del país anfitrión a la capital del } \\
\text { país de origen }\end{array}$ & Calculadora de distancia geodatos \\
\hline T_OPEN & $\begin{array}{l}\text { Costo comercial del país de origen, } \\
\text { medido como la apertura comercial } \\
\text { (Importaciones }+ \text { Exportaciones) } / \text { PIB real }\end{array}$ & $\begin{array}{l}\text { Indicadores de desarrollo económico } \\
\text { datosmacro }\end{array}$ \\
\hline
\end{tabular}

Fuente: Elaboración propia.

Es importante resaltar que en las variables IED, SUMPIB, DIFFPIB2, DIFFPIBHAB y DIST de la ecuación planteada fue necesario aplicar un logaritmo, porque presentan alta volatilidad y toman valores muy elevados.

Las primeras cuatro variables independientes de la ecuación son las variables especificas planteadas por Carr et al. (2019), el primer término SUMPIB hace referencia a la suma del producto interno bruto real del par de países, el segundo DIFFPIB2 se define como el valor absoluto de la diferencia al cuadrado del PIB real del país de origen menos el PIB real del país anfitrión. El producto interno es un indicador económico que sintetiza el nivel de actividad de una economía, siendo referente para la medición y descripción de la producción en un periodo específico de tiempo. Cuando se habla del valor total de los servicios y bienes que un país produce, se está haciendo referencia al PIB real (Morales y Antonio, 2011). En cuanto al tercer término DIFFHAB, se entiende como la diferencia de la mano de obra calificada del país de origen menos la mano de obra calificada del país anfitrión, por último, DIFFPIBHAB se define como la interacción entre la variable DIFFHAB y DIFFPIB (País de origen-País anfitrión). En el tercer y cuarto términos se tiene en cuenta la mano de obra calificada. Esta última de gran importancia en la teoría de Markusen (1984), dado que las empresas que tienen activos específicos firmes (que se desarrollan 
utilizando mano de obra calificada), tendrán mayores incentivos para expandir sus operaciones a través de las fronteras (Blonigen y Davies, 2005), es decir que son más propensas a invertir en el extranjero. También, se tuvo en cuenta la variable DIST dado que abarca criterios como transporte y otros costos comerciales que afectan la decisión de localización del inversor y es definida como la distancia medida en millas desde la capital del país anfitrión a la capital del país de origen. Finalmente, la variable T_OPEN describe la apertura comercial del país de origen, la cual es de suma importancia en el análisis, ya que mide la capacidad de las compañías para comerciar productos intermedios y finales, lo que afecta la ubicación en el extranjero de estas compañías. Para este estudio cuantitativo correlacional se tomó una muestra de 18 países como países de origen, de los cuales la mitad cuentan con un convenio vigente con Colombia en materia del impuesto de Renta. Se tuvieron en cuenta países con diferente ubicación geográfica y se clasificaron por la motivación de IED (horizontal y vertical), para identificar el signo esperado por cada variable en este estudio. En la tabla 4 se puede evidenciar los países evaluados, su ubicación, si cuenta con un convenio vigente y la motivación de IED.

Tabla 4: Reporte países evaluados, ubicación y motivación IED

\begin{tabular}{lcccc}
\hline \multicolumn{1}{c}{ País } & Ubicación & Convenio Vigente & Tipo de País & Motivación IED \\
\hline Corea & Asia & $\mathrm{Si}$ & Desarrollado & Vertical \\
China & Asia & $\mathrm{No}$ & Desarrollado & Vertical \\
Gran Bretaña & Europa & $\mathrm{Si}$ & Desarrollado & Vertical \\
Republica Checa & Europa & $\mathrm{Si}$ & Desarrollado & Vertical \\
Portugal & Europa & $\mathrm{Si}$ & Desarrollado & Vertical \\
Suiza & Europa & $\mathrm{Si}$ & Desarrollado & Vertical \\
España & Europa & $\mathrm{Si}$ & Desarrollado & Vertical \\
Bélgica & Europa & $\mathrm{No}$ & Desarrollado & Vertical \\
Italia & Europa & $\mathrm{No}$ & Desarrollado & Vertical \\
Grecia & Europa & $\mathrm{No}$ & Desarrollado & Vertical \\
Alemania & Europa & $\mathrm{No}$ & Desarrollado & Vertical \\
Dinamarca & Europa & $\mathrm{No}$ & Desarrollado & Vertical \\
México & Latinoamérica & $\mathrm{Si}$ & En desarrollo & Horizontal \\
Chile & Latinoamérica & $\mathrm{Si}$ & En desarrollo & Horizontal \\
Brasil & Latinoamérica & $\mathrm{No}$ & En desarrollo & Horizontal \\
Argentina & Latinoamérica & $\mathrm{No}$ & En desarrollo & Horizontal \\
Canadá & Norteamérica & $\mathrm{Si}$ & Desarrollado & Vertical \\
Estados Unidos & Norteamérica & $\mathrm{No}$ & Desarrollado & Vertical \\
\hline & & & & E
\end{tabular}

Fuente: Elaboración propia. 
En primera instancia se evaluaron los países desarrollados y se clasificaron dentro de la motivación vertical de IED, dado que presentan diferencias en la mano de obra calificada en comparación a Colombia, por otra parte, los países en desarrollo se clasificaron en la motivación horizontal de IED, ya que tienen unos índices de mano de obra calificada similar a la de Colombia. Con base a esto, los signos de los coeficientes esperados por cada variable según la motivación son los presentados en la tabla 5.

Tabla 5: Signo esperado por variable

\begin{tabular}{lcc}
\hline \multirow{1}{*}{ Variable } & \multicolumn{2}{c}{ Resultado esperado } \\
& Motivación vertical & Motivación horizontal \\
\hline SUMPIB $(\log )$ & + & + \\
DIFFPIB2 $(\log )$ & - & + \\
DIFFHAB & + & - \\
DIFFPIBHAB $(\log )$ & - & + \\
T_OPEN & + & - \\
\hline
\end{tabular}

Fuente: Elaboración propia.

Para el análisis estadístico se utilizó la técnica de mínimos cuadrados ordinarios (MCO), la cual a través de una regresión lineal múltiple permite estimar de la manera más adecuada los posibles coeficientes de cada término de la ecuación que mide la IED, a partir de parámetros muestrales (18 países). El objetivo de este procedimiento estadístico es encontrar la mejor combinación de parámetros (variables independientes) que minimicen la suma de cuadrados, es decir el error de estimación, el cual hace referencia a la parte de la variable dependiente que no se pudo explicar con las variables independientes; este error se describe matemáticamente como la diferencia entre la variable calculada y la variable observada (Martínez Bencardino, 2012). Este método estadístico fue seleccionado dentro de este estudio, dado que permite encontrar los mejores estimadores lineales libres de sesgo, a partir de la reducción del error en su máxima expresión. Adicionalmente es una técnica de aplicación no tan compleja y se adecúa a los supuestos de los modelos econométricos, generando un resultado más propicio orientado a la realidad actual (Blonigen y Davies, 2004).

\section{Resultados}

En esta sección del documento se presentan los resultados obtenidos a partir de una regresión lineal múltiple, que busca medir el efecto de los convenios de doble imposición en la inversión extranjera directa en Colombia. En el análisis econométrico planteado se aplicaron 2 regresiones lineales 
múltiples, la primera tomando los países que actualmente tienen un convenio de doble imposición vigente en materia de impuesto sobre la renta con Colombia y la otra con países que no tienen un convenio vigente; por cada regresión se tomó una muestra total de 9 países.

En la tabla 6 se observa la tabla de frecuencia por tipo de país y ubicación geográfica. De los 18 países, el $78 \%$ corresponde a países desarrollados y el $22 \%$ a países en vía de desarrollo, de igual manera se observa que el $56 \%$ de estos países están ubicados en Europa, el $22 \%$ en Latinoamérica, el $11 \%$ en Asia y el $11 \%$ restante en Norteamérica.

Tabla 6. Tabla de frecuencia por tipo de país y ubicación geográfica

\begin{tabular}{lccc}
\hline \multicolumn{1}{c}{ Clasificación } & N & Frecuencia absoluta & Frecuencia porcentual \\
\hline Tipo de país & 18 & & \\
Países desarrollados & & 14 & $78 \%$ \\
Países en desarrollo & & 4 & $22 \%$ \\
Ubicación & 18 & & \\
Asia & & 2 & $11 \%$ \\
Europa & & 4 & $56 \%$ \\
Latinoamérica & & 2 & $22 \%$ \\
Norteamérica & & & $11 \%$ \\
\hline
\end{tabular}

Fuente: Elaboración propia.

En la tabla 7 se observan los estadísticos descriptivos de la regresión con convenio. Se puede observar que los datos de las variables se encuentran muy dispersos frente a su media, ya que, se considera que si superan el $50 \%$ presentan una alta desviación (Perez Vicente, s.f). La variable dependiente (IEDb) y las variables independientes DIFFPIB2 y DIFFPIHAB presentan una alta desviación debido a las diferencias económicas entre los países observados (desarrollados y en desarrollo).

Tabla 7: Estadísticos descriptivos regresión con convenio

\begin{tabular}{lcccc}
\hline \multicolumn{1}{c}{ Variable } & Media & Desviación & $C V$ & $N$ \\
\hline IEDb $(\log )$ & 4.3314 & .5771 & .1332 & 9 \\
SUMPIB $(\log )$ & 6.0783 & .2700 & .0444 & 9 \\
DIFFPIB2 $(\log )$ & 11.3183 & 1.1377 & .1005 & 9 \\
DIFFHAB & .2101 & .1122 & .5341 & 9 \\
DIFFPIBHAB $(\log )$ & 4.962 & .6129 & .1235 & 9 \\
DIST $(\log )$ & 3.6376 & .1971 & .0542 & 9 \\
T_OPEN & .7063 & .3315 & .4694 & 9 \\
\hline
\end{tabular}

Fuente: Elaboración propia. 
Sora, B., y Bohorquez, N. Convenios de doble imposición del impuesto de renta...

En la tabla 8 se observan los estadísticos descriptivos de la regresión sin convenio. A través del coeficiente de variación se determinó que la mayoría de los datos de cada variable son casi heterogéneos y presentan una alta volatilidad. Esto es debido a que los países observados presentan grandes diferencias en los indicadores de desarrollo económico (PIB, mano de obra calificada y apertura comercial). Los indicadores de desarrollo miden el crecimiento económico de un país agregando variables económicas y sociales. Esto permite tomar decisiones respecto a las necesidades nacionales internas e internacionales a través de políticas, programas y proyectos gubernamentales (Guillén Badii, Garza y Acuña, 2015). Los resultados muestran que los coeficientes son directamente proporcionales, es decir que entre más diferencia exista entre la economía de cada país, más alta será la desviación, ya que los datos se encuentran muy dispersos frente a su media (Pérez Vicente, s.f).

Tabla 8: Estadísticos descriptivos regresión sin convenio

\begin{tabular}{lcccc}
\hline \multicolumn{1}{c}{ Variable } & Media & Desviación & $C V$ & $N$ \\
\hline IEDb $(\log )$ & 4.2962 & .8696 & .2024 & 9 \\
SUMPIB $(\log )$ & 6.3224 & .5075 & .0803 & 9 \\
DIFFPIB2 $(\log )$ & 11.7801 & 1.8987 & .1611 & 9 \\
DIFFHAB & .2781 & .1920 & .6904 & 9 \\
DIFFPIBHAB $(\log )$ & 4.962 & .6129 & .1235 & 9 \\
DIST $(\log )$ & 5.0254 & 1.0069 & .2003 & 9 \\
T_OPEN & .5363 & .4396 & .8196 & 9 \\
\hline
\end{tabular}

Fuente: Elaboración propia.

En la tabla 9 se observa un resumen de los 2 modelos planteados donde se indica la correlación entre las variables independientes y la variable dependiente (IEDb). Para el caso de la primera regresión el coeficiente de correlación múltiple es .982 , es decir que el $96,4 \%$ de la variabilidad de la inversión extranjera directa en Colombia está explicada por las variables independientes planteadas en el modelo CMM. Para el caso de la regresión de los países que no cuentan con un convenio vigente el coeficiente de correlación múltiple es .930 , donde el $86,5 \%$ de la variabilidad del factor dependiente está explicada por las variables independientes. Con base a lo anterior, en los dos modelos se observa un alto porcentaje de explicación de las variables independientes; sin embargo, esto no significa que estas variables influyan de manera absoluta en el comportamiento de la inversión extranjera en Colombia. Se esperaría que el análisis de los países sin convenio presentase un coeficiente de correlación bajo, por lo que deben existir otras variables no identificadas en el estudio que están afectando la inversión extranjera. 
Tabla 9: Resumen de los modelos

\begin{tabular}{lcccc}
\hline \multicolumn{1}{c}{ Modelo } & R & R2 & R2 ajustado & Error estándar de la estimación \\
\hline Con convenio & $.982 \mathrm{a}$ & .964 & .892 & .18947 \\
Sin convenio & $.930 \mathrm{a}$ & .865 & .595 & .55325 \\
\hline
\end{tabular}

a. Predictores: (Constante), SUMPIB, DIFFPIB2, DIFFHAB, DIFFPIBHAB, DIST, T_OPEN

Fuente: Elaboración propia.

En la tabla 10 se observan los coeficientes $\beta$ y los signos esperados por cada variable. Para establecer los signos esperados detallados en la tabla $10 \mathrm{y}$ evaluar la correlación de manera más objetiva se tuvo en cuenta la motivación vertical de IED de acuerdo con el método CMM. Con base a los datos obtenidos en la tabla 6 , el $78 \%$ de los países analizados son países desarrollados, lo cual puede influir en los resultados obtenidos ya que los países inversores no son comparables con un país como Colombia. Dicha comparación no es posible ya que los países desarrollados tienen diferentes niveles de tecnología, información, aprendizaje, capacidades y experiencia de los trabajadores y cooperación, entre otras, en comparación a los países en vía de desarrollo (Vélez y González, 2008).

Tabla 10: Coeficientes de regresión

\begin{tabular}{lccc}
\hline \multirow{2}{*}{ Variable } & Países con convenio & Países sin convenio & $\begin{array}{c}\text { Signo } \\
\text { esperado }\end{array}$ \\
\cline { 2 - 3 } & \multicolumn{2}{c}{ Coeficiente $\boldsymbol{\beta}$} & + \\
\hline SUMPIB $(\log )$ & $-3,425$ & 2,916 & - \\
DIFFPIB2 $(\log )$ & $-0,304$ & $-0,039$ & + \\
DIFFHAB & $-3,968$ & 0,908 & - \\
DIFFPIBHAB $(\log )$ & 2,063 & $-0,129$ & - \\
DIST $(\log )$ & $-0,269$ & $-0,645$ & + \\
T_OPEN & $-1,797$ & 1,333 & + \\
\hline
\end{tabular}

Fuente: Elaboración propia.

Con base en los datos estadísticos obtenidos en la tabla 10 la variable SUMPIB (suma de los PIB de los 2 países), si las otras variables se mantienen constantes, presenta en la regresión con convenio una relación inversa (el aumento de una unidad en la suma de los PIB disminuye en promedio el valor de la inversión extranjera en 3.425) y en la regresión sin convenio una relación positiva (el aumento de una unidad en la suma de los PIB con la variable dependiente incrementa en promedio 2.916).

El signo esperado en la variable SUMPIB es positivo, dado que se espera que los países con economías más grandes motiven a las empresas a invertir fuera 
Sora, B., y Bohorquez, N. Convenios de doble imposición del impuesto de renta...

de sus fronteras, específicamente en países en desarrollo. Esto implica que, al ser países con diferencia en tamaño y economía (desarrollada y en desarrollo), se espera una relación positiva (los países con un PIB alto son más atraídos a invertir en el extranjero).En cuanto a la variable DIFFPIB2 (diferencia de los PIB al cuadrado), presenta en las dos regresiones una relación negativa con la variable dependiente, lo que indica que, si las otras variables se mantienen constantes, el aumento de una unidad de DIFFPIB2 disminuye en promedio el valor de la inversión extranjera en .304 en la regresión con convenio y en .039 en la regresión sin convenio. Esto puede ser consecuencia de las diferencias en tamaño y economía de los países evaluados. De igual forma, la variable DIFFHAB (diferencia de las habilidades de población), presenta en la regresión con convenio una relación inversa y en la regresión sin convenio una relación directa con la variable dependiente. El aumento de una unidad en DIFFHAB disminuye en promedio el valor de la inversión extranjera en 3.968 en la primera regresión e incrementa en promedio .908 en la segunda regresión cuando las otras variables se mantienen constantes. El signo esperado en esta variable es positivo, dado que los países desarrollados buscan invertir en países en desarrollo para ahorrar costos en la mano de obra. La variable DIFFPIBHAB (interacción diferencia PIB y diferencia habilidades), presenta en la primera regresión una relación positiva y en la segunda regresión una relación inversa con la variable dependiente. Por lo tanto, si las otras variables se mantienen constantes, el aumento de una unidad de DIFFPIBHAB incrementa en promedio el valor de la inversión extranjera en 2.063 y disminuye en promedio .121 en países con y sin convenio respectivamente. Al igual que la variable DIFFPIB esto está dado por la diferencia en tamaño y economía de los países evaluados, los signos opuestos en las regresiones pueden ser consecuencia de que los países sin convenio presentan mayores diferencias que los con convenio. Adicionalmente, la variable DIST (distancia entre países), presenta una relación negativa con la variable dependiente en el caso de países con y sin convenio. Lo anterior indica que, si las otras variables se mantienen constantes, el aumento de una unidad en la distancia medida en millas entre las capitales disminuye en promedio el valor de la inversión extranjera (.269 en países con convenio y en .645 en países sin convenio). Esto es congruente con la amplia distancia en millas que presentan las capitales de los países evaluados frente a Bogotá, la capital de Colombia, ya que la localización es un factor que afecta la decisión del inversor. Finalmente, la variable T_OPEN (apertura comercial del país origen) presenta en los países con convenio (primer caso) una relación inversa y en los países sin convenio (segundo caso) una relación directa con la variable dependiente. La apertura comercial es conocida también como liberalización comercial y elimina la discriminación de la política comercial entre el mercado nacional y exterior (Alonso y Garcimartín, 2005). Los resultados señalaron 
que el aumento de una unidad en los costos comerciales del país anfitrión disminuye en promedio el valor de la inversión extranjera en 1.797 en el primer caso e incrementa en promedio 1.333 en el segundo caso, cuando las otras variables se mantienen constantes. Lo anterior puede ser consecuencia de que los países evaluados presentan grandes diferencias en la apertura comercial, puesto que se esperaría que el coeficiente fuera positivo, dado que los países con mayor apertura comercial deciden expandir sus operaciones fuera de sus fronteras en mayor medida que los países con una apertura comercial menor. Según los resultados mostrados en la tabla 10 se define el modelo de la investigación de la siguiente manera:

$$
I E D_{b}=f(-3.425,-0.304,-3.968,2.063,-0.269,-1.797)
$$

Finalmente, en base a los resultados obtenidos, donde los países sin convenio muestran relación con la inversión extranjera, se rechaza la hipótesis planteada. Adicionalmente, a pesar de encontrar un alto grado de relación entre las variables independientes y la inversión extranjera directa, se observó que los signos previstos para el modelo no se cumplieron a cabalidad, en el caso de la regresión con convenio solo 2 de las 6 variables cumplieron con el signo esperado. Sin embargo, en la regresión sin convenio las 6 variables cumplieron con el signo previsto, a pesar de que algunos de los coeficientes no tomaron un valor significativo.

\section{Conclusiones}

Las investigaciones de Barthel et al. (2010), Blonigen y Davies (2004), Blonigen et al. (2014), Castillo y López (2019), Braun (2016), Marques y Pinho (2014), Murthy y Bhasin (2015) y Neumayer (2007) abordadas previamente en la revisión literaria, concluyeron que los convenios de doble imposición tienen un efecto positivo en la inversión extranjera. Sin embargo, el análisis de datos del presente estudio contradice los resultados de dichas investigaciones ya que se observó que los convenios no tienen ningún efecto en la inversión extranjera directa, debido a que, se observa un nivel alto de relación en países que no cuentan con un convenio vigente, lo cual puede estar explicado por factores no observados en el modelo.

Los resultados de esta investigación fueron congruentes con el estudio de Baker (2014), quien no encontró efecto entre las dos variables estudiadas. Los datos mostraron que los tratados de doble tributación de renta no implican el aumento de la inversión extranjera directa en Colombia, y la falta de un efecto estadísticamente significativo en la regresión convenio se interpreta como una prueba de que no existe relación entre las 2 variables. Futuros estudios podrían analizar las ventajas tributarias de la firma de los convenios de doble imposición en Colombia, identificando los costos de entrada y otras variables que logren 
Sora, B., y Bohorquez, N. Convenios de doble imposición del impuesto de renta...

un incremento de la inversión extranjera, junto con su tasa de recuperación, la cual puede ser fundamental para la inversión extranjera directa. Ya que actualmente el gobierno colombiano adelanta una estrategia para contrarrestar la crisis actual del país promoviendo la inversión extranjera directa (Revista El Congreso, 2020), futuros estudios también podrían abordar el efecto que estos nuevos incentivos tributarios y la simplificación de trámites de localización de multinacionales asiáticas y estadounidenses tienen sobre la inversión extranjera directa. Este estudio presentó limitantes con relación a la muestra de países, ventana de observación y tipo de impuesto. Investigaciones futuras podrían analizar la relación entre los convenios de doble imposición con una muestra más amplia de países (incluyendo países más similares a Colombia) y un periodo de tiempo más amplio, incluso examinando impuestos diferentes al de renta. Adicionalmente, es relevante evaluar en un futuro el comportamiento de las variables económicas antes y después de la firma de un convenio y abordar países que presenten características similares a las de Colombia, para poder determinar la relación de los convenios en la motivación horizontal de IED.

\section{Referencias bibliográficas}

Acosta Rodríguez, D. (2013). Generalidades del impuesto de renta. En Acosta Rodríguez, D., Impuesto de renta (pp. 6-12). Bogotá: Kimpres Ltda.

Alonso, J. A. y Garcimartín, C. (2005). Apertura comercial y estrategia de desarrollo. Baker, P. (2014). An Analysis of Double Taxation Treaties and their Effect on Foreign Direct Investment. International Journal of the Economics of Business, 21(3), 341-377. DOI: 10.1080/13571516.2014.968454

Baker, P. L. (2014). An analysis of double taxation treaties and their effect on foreign direct investment. International Journal of the Economics of Business, 21(3), 341-377.

Barthel, F., Busse, M., \& Neumayer, E. (2010). The impact of double taxation treaties on foreign direct investment: evidence from large dyadic panel data. Contemporary Economic Policy, 28(3), 366-377. DOI: 10.1111/j.1465-7287.2009. 00185.x

Blonigen, B., \& Davies, R. (2004). The Effects of Bilateral Tax Treaties on U.S. FDI Activity. International Tax and Public Finance, 11(5), 601-622. DOI: 10.1023/b: itax.0000036693.32618.00

Blonigen, B., \& Davies, R. (2005). Do Bilateral Tax Treaties Promote Foreign Direct Investment? NBER Working Papers, 1-1.

Blonigen, B. A., Oldenski, L., \& Sly, N. (2014). The differential effects of bilateral tax treaties. American Economic Journal: Economic Policy, 6(2), 1-18.

Braun, J. (2016). The effects of double tax treaties for developing countries. A case study of Austria's double tax treaty network. Public Finance \& Management, 16(4), 383-433.

Bruce, A., Blonigen, L., \& Sly, N. (2014). The Differential Effects of Bilateral Tax Treaties. American Economic Journal: Economic Policy, 6(2), 1-18.

Carr, D., Markusen, J., \& Maskun, K. (2003). Estimating the Knowledge-Capital Model of the Multinational Enterprise: Reply. The American Economic Review. 93(3), 995-1001. 
Castillo, A., \& López, J. (2019). Efecto de los Tratados de Doble Imposición sobre la actividad de Inversión Extranjera Directa bilateral española. Economics: The OpenAccess, Open-Assessment E-Journal, 13, 19-22.

Davies, R. (2003). Tax Treaties, Renegotiations, and Foreign Direct Investment. In Economic Analysis and Policy, 33(2), 251-273. DOI: 10.1016/S0313-5926(03)50020-0

Dirección de Impuestos y Aduanas Nacionales [DIAN]. (2019). Convenios tributarios internacionales: Acuerdos para eliminar la doble tributación en Materia de Impuestos sobre la Renta. Recuperado de: https://www.dian.gov.co/

Duque Romero, L. (2009). Métodos para eliminar la doble imposición internacional en Colombia. Revista de Derecho Fiscal: Universidad Externado de Colombia. (5).

Guillen, A., Badii, M. H., Garza, F., \& Acuña, M. (2015). Descripción y Uso de Indicadores de Crecimiento Económico. Revista Daena (International Journal of Good Conscience), 10(1).

Marketers by Adlatina. (2019). Los países de América más atractivos para invertir: EE. UU., Brasil y Canadá. Recuperado de: http://www.marketersbyadlatina.com/

Marqués, M., \& Pinho, C. (2014). Tax-Treaty Effects on Foreign Investment: Evidence from European Multinationals. FinanzArchiv / Public Finance Analysis, 70(4), 527-555

Martínez Bencardino, C. (2012). Regresión y Correlación. En Martínez Bencardino, C., Estadística y muestreo (pp. 637-647). Bogotá: Ecoe Ediciones.

Morales, P., y Antonio, J. (2011). Estimación y análisis del producto interno bruto potencial de los municipios del estado de Quintana Roo, 2003-2007 (Master's thesis, Universidad de Quintana Roo).

Murthy, K., \& Bhasin, N. (2015). The impact of bilateral tax treaties: A multi-country analysis of FDI inflows into India. Journal of International Trade \& Economic Development, 24(6), 751-766. DOI: 10.1080/09638199.2014.960442

Neumayer, E. (2007). Do double taxation treaties increase foreign direct investment to developing countries? Journal of Development Studies, 43(8), 1501-1519. DOI: $10.1080 / 00220380701611535$

Ocampo Herrán, C. (2000). La doble tributación internacional, principios y realidades de los convenios. (Tesis de maestría). Pontificia Universidad Javeriana, Bogotá, Colombia.

Organización para la cooperación y el desarrollo económico [OCDE]. (2010). Modelo de Convenio Tributario sobre la Renta y sobre el Patrimonio. [Traducido al español de Model Tax Convetion on Income and on Capital condensed version]. España: Instituto de Estudios Fiscales (IEF) para edición español.

Pérez Vicente, S. (s.f). Estadística Básica. Aplicación con SPSS. (Tesis de doctorado). APES Hospital Costa del Sol, Marbella, España.

Vélez, M. A. y González, C. B. (2008). Conocimiento y crecimiento económico: una estrategia para los países en vías de desarrollo. Revista de Economía mundial, (18), 65-77. 